\title{
Análisis de costo efectividad de prasugrel, comparado con clopidogrel para pacientes con síndrome coronario agudo sometidos a intervención coronaria percutánea en Colombia
}

\author{
Analysis of cost effectiveness of prasugrel compared \\ with clopidogrel for patients with acute coronary \\ syndrome undergoing percutaneous coronary \\ intervention in Colombia
}

\author{
Aurelio Mejía • (Bogotá, D.C.), Juan Manuel Senior, Mateo Ceballos, \\ Sara Atehortúa, Juan Manuel Toro, Clara Inés Saldarriaga, María \\ Elena Mejía, Carolina Ramírez • Medellín (Colombia)
}

\section{Resumen}

Objetivo: determinar, desde la perspectiva del sistema de salud colombiano, la relación de costo-efectividad del prasugrel comparado con clopidogrel, para el tratamiento de pacientes adultos con síndrome coronario agudo.

Material y métodos: se construyó un modelo de Markov con ciclos anuales en el cual los pacientes pueden permanecer sin experimentar nuevos eventos cardiovasculares, sufrir un nuevo evento o morir. En el caso base se adoptó un horizonte temporal de 10 años y una tasa de descuento de 3\%. Las probabilidades de transición se extrajeron del ensayo clínico TRITON-TIMI 38, de las estadísticas vitales del Departamento Nacional de Estadística y de la información de los pacientes colombianos del registro ACCESS. Para identificar y medir el uso de recursos se diseñó un caso típico a partir de la revisión de guías y protocolos; para la valoración se emplearon manuales tarifarios colombianos. Se realizaron análisis de sensibilidad determinísticos y probabilísticos.

Resultados: en el caso base, el costo por año de vida ajustado por calidad ganado con prasugrel es \$79 987695 pesos colombianos. Los resultados son sensibles a cambios en el horizonte temporal y al costo del clopidogrel. Bajo un umbral de disposición a pagar de tres veces el PIB per cápita colombiano, la probabilidad de que el prasugrel sea costo efectivo es $7 \%$.

Conclusiones: la decisión respecto a la inclusión del prasugrel en el tratamiento de pacientes con síndrome coronario agudo, sometidos a intervención coronaria percutánea depende fundamentalmente del costo del clopidogrel que el decisor considere relevante para realizar la comparación. (Acta Med Colomb 2015; 40: 310-317).

Palabras clave: economía médica, costos y análisis de costos, economía de la salud, estudios de evaluación como asunto, prasugrel, clopidogrel, Colombia.

\footnotetext{
Abstract

Objective: to determine the cost-effectiveness relation of prasugrel compared with clopidogrel for the treatment of adult patients with acute coronary syndrome from the perspective of Colombian health system.

Material and methods: a Markov model with annual cycles in which patients can remain without experiencing new cardiovascular events, have a new event or die, was built. In the base case a time horizon of 10 years and a discount rate of $3 \%$ was adopted. Transition probabilities were taken from the clinical trial TRITON-TIMI 38, of vital statistics from the National Department of Statistics and from the information of Colombian patients in ACCESS registry. To identify and measure the use of resources, a typical case was designed from the review of guidelines and protocols; Colombian tariff manuals were used for assessment. Deterministic and probabilistic sensitivity analyzes were performed.
}

Sr. Aurelio Mejía: Economista, MSc en Economía de la Salud. Instituto de Evaluación Tecnológica en Salud-IETS (Bogotá, D.C.); Dr. Juan Manuel Senior, FACP: Especialista en Medicina Interna y Cardiología Clínica, Subespecialista en Cardiología Intervencionista, Hemodinamia y Vascular Periférico. Coordinador Cardiología Clínica y Cardiología Intervencionista, Facultad de Medicina, Universidad de Antioquia. Grupo de Rehabilitación en Salud; Sr. Mateo Ceballos González: Economista. Grupo de Economía de la Salud, Facultad de Ciencias Económicas, Universidad de Antioquia; Sra. Sara Atehortúa: Economista, MSc en Gobierno y Asuntos Públicos. Departamento de Economía, Facultad de Ciencias Económicas, Universidad de Antioquia; Dr. Juan Manuel Toro: Especialista en Medicina Interna, MSc en Epidemiología. Departamento de Medicina Interna, Facultad de Medicina, Universidad de Antioquia. Grupo Académico de Epidemiología Clínica; Dra. Clara Inés Saldarriaga: Especialista en Medicina Interna y Cardiología, Clínica Cardiovascular. Profesora del Departamento de Cardiología, Facultad de Medicina, Universidad de Antioquia; Sras. María Elena Mejía y Carolina Ramírez. Economistas. Grupo de Economía de la Salud, Facultad de Ciencias Económicas, Universidad de Antioquia. Medellín (Colombia).

Correspondencia. Sr. Mateo Ceballos, Bogotá D.C. (Colombia)

E-mail: mateoceballosgo@gmail.com Recibido: 14/VIII.2015 Aceptado: 21/X/2015 
Results: in the base case, the cost per year of quality-adjusted life gained with prasugrel is $\$$ $79,987,695$ Colombian pesos. The results are sensitive to changes in the timeframe and cost of clopidogrel. Under a threshold willingness to pay three times the per capita GDP of Colombia, the probability that prasugrel may be cost-effective, is $7 \%$.

Conclusions: the decision on the inclusion of prasugrel in the treatment of patients with acute coronary syndrome undergoing percutaneous coronary intervention depends mainly on the cost of clopidogrel that the decision maker considers relevant to perform the comparison. (Acta Med Colomb 2015; 40: 310-317).

Keywords: health economics, costs and cost analysis, health economics, evaluation studies as subject, prasugrel, clopidogrel, Colombia.

\section{Introducción}

El síndrome coronario agudo (SCA) es una de las emergencias médicas más frecuentes en países en vía de desarrollo y, dentro de las enfermedades cardiovasculares, es la manifestación más prevalente (1). En Colombia, según estadísticas vitales 2007-2008 (2) la tasa de mortalidad por enfermedades isquémicas del corazón en ese mismo periodo, para mayores de 45 años fue de 263.7 x 100000 habitantes. La expectativa de vida en la población colombiana proyecta un aumento de 67.8 años de vida entre 1985 y 1990 a 73.9 años entre el 2010 y 2015 , es decir, durante este periodo la población mayor de 45 años se triplicará, con lo cual la incidencia y la prevalencia de la enfermedad cardiaca aterosclerótica también aumentará, con el consecuente incremento en el costo de atención en salud.

De otro lado y según las estadísticas publicadas por el Departamento Administrativo Nacional de Estadísticas, DANE, de acuerdo con la clasificación internacional de enfermedades CIE-10, la enfermedad isquémica cardiaca fue la principal causa de muerte en personas mayores de 55 años, por encima del cáncer y la agresión. La alta morbilidad y mortalidad de esta enfermedad plantean un reto importante debido al impacto que producen en el estado de salud, en la calidad de vida, y en los aspectos sociales, laborales y económicos del país.

El SCA genera altos costos sociales, ocasionados por su tratamiento y por las pérdidas de productividad de quienes lo padecen. Se han realizado múltiples estudios para determinar los tratamientos más costo efectivos y la duración óptima de los mismos, en los cuales se ha encontrado que los individuos que reciben el tratamiento estándar con aspirina después de un evento coronario agudo permanecen con alto riesgo de sufrir eventos coronarios en el corto y largo plazo (3-6). Por ello, se han desarrollado medicamentos como el clopidogrel, el cual ha demostrado que, adicionado al tratamiento convencional con aspirina, mejora la calidad de vida de los pacientes y reduce el riesgo de nuevos eventos cardiovasculares $(7,8)$.

El reciente estudio TRITON TIMI 38 (9) evaluó la seguridad y eficacia del prasugrel en los pacientes con SCA, incluyendo 13608 pacientes que se llevaron a angiografía coronaria, y cuya anatomía coronaria era conocida. Se comparó la administración de prasugrel dosis de carga de $60 \mathrm{mg}$ seguido de $10 \mathrm{mg} /$ día contra clopidogrel $300 \mathrm{mg}$ seguido de $75 \mathrm{mg} /$ día. El estudio excluyó a los pacientes que habían recibido clopidogrel en los cinco días previos al ingreso. Los resultados mostraron una disminución en el desenlace compuesto de muerte de causas cardiovasculares, infarto o evento cerebrovascular no fatal a 30 días a favor de prasugrel (9.9\% vs $12.1 \%$ HR 0.8 IC $0.73-0.90$ ) y al analizar por separado cada uno de los desenlaces se encontró una disminución significativa en el infarto no fatal, en la revascularización urgente del vaso culpable y en la trombosis del stent.

Los desenlaces de seguridad mostraron que el grupo asignado a prasugrel tuvo un incremento en el sangrado mayor según la definición TIMI (2.4\% vs $1.8 \%$ HR 1.32 IC 1.03-1.68) y en el sangrado que amenaza la vida, pero sin diferencias en el sangrado intracraneal. El sangrado fue más frecuente en los pacientes con peso menor de 60 kilos, edad mayor a 75 años y con antecedente de accidente cerebrovascular o isquemia cerebral transitoria previa. También se realizó un subanálisis del grupo de pacientes diabéticos en quienes se observó una mayor eficacia, con reducción relativa del riesgo de infarto de $40 \%$ sin incrementar el sangrado, con un beneficio neto a favor de prasugrel.

Lo anterior plantea una disyuntiva acerca de cuál alternativa debería implementarse en el sistema de salud colombiano, con el fin de elegir la estrategia que ofrezca los mayores beneficios en salud, considerando las restricciones presupuestales del país. Teniendo en cuenta lo anterior, el objetivo de este artículo es evaluar, desde la perspectiva del sistema de salud colombiano, el tratamiento con aspirina más prasugrel, comparado con aspirina más clopidogrel, para pacientes con SCA sometidos a intervención coronaria percutánea (ICP) en Colombia.

\section{Metodología}

Análisis de costo-utilidad desde la perspectiva del sistema de salud colombiano. La población corresponde a pacientes mayores de 18 años con SCA, con y sin elevación del segmento ST. Las opciones consideradas son: 
1. Aspirina más clopidogrel: esta es la estrategia que corresponde a la práctica actual en el sistema colombiano. Carga inicial de $300 \mathrm{mg} ; 75 \mathrm{mg}$ diarios durante un año.

2. Aspirina más prasugrel: carga inicial de $60 \mathrm{mg} ; 10 \mathrm{mg}$ diarios durante un año.

En adelante, cuando se emplee el término clopidogrel o prasugrel deben entenderse como la terapia de antiagregación dual.

Para estimar los costos y la efectividad de ambas intervenciones se planteó un modelo de Markov en el cual se contemplan cuatro desenlaces independientes en el primer año: infarto del miocardio (IM), accidente cerebrovascular $(\mathrm{ACV})$, ningún evento y muerte. Los pacientes que experimentan un IM o ACV pueden fallecer o progresar a los estadios pos IM o pos ACV. Estos dos últimos estadios permiten capturar los costos y desenlaces de los pacientes que experimentaron algún evento no fatal durante el primer año de seguimiento o en alguno de los años posteriores.

El modelo incluye dos estadios "túnel”, IM y ACV. Estos estadios se diferencian de los estadios pos IM y pos ACV en la medida en que los pacientes sólo permanecen un ciclo en dicho estadio (cuando experimentan alguno de los eventos), de manera que al año siguiente los pacientes progresan a pos IM, pos ACV o mueren. Sin embargo, es probable que un paciente que sufrió un reinfarto durante el primer año pueda experimentar un nuevo IM en los años posteriores. Por lo tanto para tener en cuenta esta posibilidad, en un análisis de sensibilidad se modificó la estructura del modelo para incluir la probabilidad de experimentar nuevos eventos coronarios en el largo plazo.

Finalmente, el estadio "No evento" captura los pacientes que no experimentan un nuevo evento. A medida que transcurre la simulación, los pacientes que permanecen en este estadio están en riesgo de experimentar un nuevo evento. Adicionalmente, el modelo incluye la probabilidad de sangrado mayor (según la definición empleada en el estudio TRITON-TIMI 38).

Se asume, teniendo en cuenta los modelos publicados y el hecho de que los ensayos clínicos tuvieron un seguimiento inferior a un año, que no existen diferencias en el riesgo de estos eventos después del primer año, lo cual significa que el efecto de las estrategias en el largo plazo está determinado, fundamentalmente, por el efecto que tengan en los desenlaces al primer año (Figura 1).

Las probabilidades de transición se estimaron a partir de diversas fuentes (Tabla 1). En primer lugar, se realizó una revisión sistemática en la base de datos Cochane, con el fin de establecer la efectividad de las intervenciones al primer año. El protocolo de búsqueda de la revisión sistemática se presenta a continuación:

((Acute Coronary Syndrome[Mesh] OR Acute Coronar*[tiab] OR Coronary Syndrom*[tiab] OR STEMI[tiab] OR non-STEMI[tiab]) AND (systematic review[tiab] OR Meta-Analysis[pt] OR Meta-Analysis as Topic[mh] OR Meta-Analys*[tiab] OR "Cochrane Database

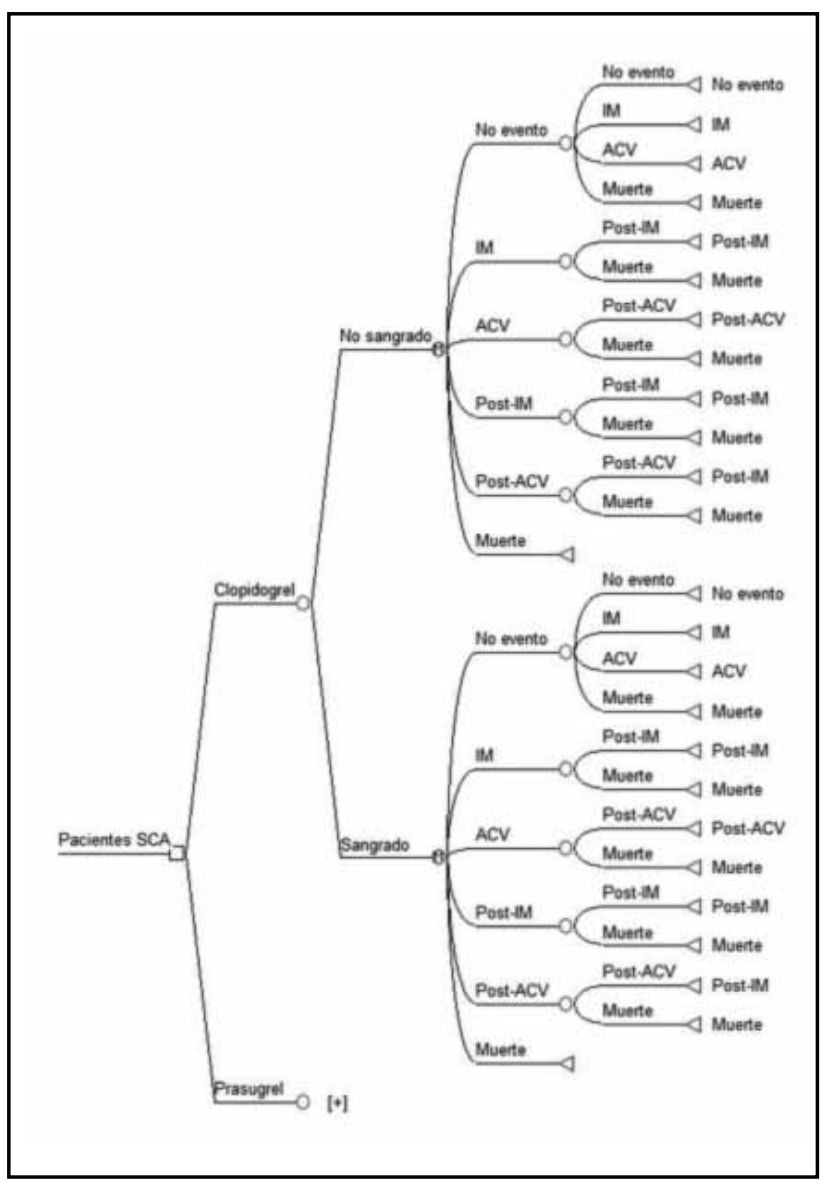

Figura 1. Modelo de decisiones para la comparación entre prasugrel y clopidogrel.

Syst Rev"[Journal:_jrid21711])) AND ((Aspirin[Mesh] OR Acetylsalicylic Acid[tiab] OR aspirin*[tiab] OR ASA[tiab]) AND (clopidogrel[all] OR prasugrel[all])).

La probabilidad de muerte (ajustada por edad) se estimó a partir de las estadísticas vitales del Departamento Administrativo Nacional de Estadística de Colombia, DANE. El riesgo de nuevos eventos coronarios en el largo plazo se estimó a partir de los datos de pacientes colombianos incluidos en el registro ACCESS. Las ponderaciones de utilidad para cada uno de los desenlaces se tomaron del estudio PLATO HECON realizado por AstraZeneca (9) (si bien este estudio se realizó para evaluar el ticagrelor, la población corresponde a pacientes con SCA, e incluyó pacientes colombianos, por lo tanto se considera que las ponderaciones de utilidad de dicho estudio pueden emplearse en esta evaluación económica).

La identificación de los eventos generadores de costos, partió de la especificación de las alternativas por comparar y del modelo de decisiones. En este paso se incluyeron los recursos con mayor impacto en los costos y por ende con mayor probabilidad de influir en la decisión, como la hospitalización en la unidad de cuidados intensivos, la elección del tipo de stent (medicado o convencional) para la intervención coronaria percutánea, los programas de 
Tabla 1. Información de efectividad utilizada en los modelos.

\begin{tabular}{|l|c|c|}
\hline Variable & Valor & Fuente \\
\hline \multicolumn{3}{|c|}{ Probabilidades de transición } \\
\hline Probabilidad de IM al primer año prasugrel & 0.0697 & Wiviott et al. (10) \\
Probabilidad de ACV al primer año prasugrel & 0.0090 & \\
Probabilidad de sangrado mayor & 0.0217 & \\
Probabilidad de muerte al primer año prasugrel & 0.0195 & \\
Probabilidad de IM al primer año clopidogrel & 0.0912 & \\
Probabilidad de ACV al primer año clopidogrel & 0.0088 & \\
Probabilidad de sangrado mayor clopidogrel & 0.0165 & \\
Probabilidad de muerte al primer año clopidogrel & 0.0221 & \\
\hline Probabilidad de IM en el largo plazo & 0.031 & Datos colombianos \\
Probabilidad de ACV en el largo plazo & 0.015 & del registro ACCESS \\
\hline \multicolumn{2}{|c|}{ Ponderaciones de utilidad } \\
\hline Riesgo relativo para ajustar mortalidad \\
\hline Post IM & 0.842 & AstraZeneca (9) \\
ACV evento & 0.779 & \\
\hline RR mortalidad después de ACV largo plazo & 0.703 & \\
\hline RR mortalidad después de IM largo plazo & 0.821 & \\
\hline
\end{tabular}

rehabilitación cardiaca, medicamentos e insumos, entre otros. La medición de los recursos consumidos en cada uno de los desenlaces se realizó mediante la construcción de un caso típico a partir de la revisión de guías de práctica clínica y protocolos de atención de un hospital universitario colombiano. Estos resultados fueron discutidos con varios especialistas en medicina interna y cardiología, y mediante consenso informal se estimó la frecuencia de uso de servicios por cada opción de manejo clínico.

El costo unitario de los medicamentos se obtuvo del Sistema de Información de Precios de Medicamentos, SISMED, del Ministerio de Salud y Protección Social de Colombia (11). El dato corresponde al precio ponderado (según número de unidades vendidas) de las diferentes presentaciones del medicamento en el mercado colombiano, el cual comprende tanto los medicamentos genéricos como las moléculas originales. Además se tuvo en cuenta en el análisis de sensibilidad el precio máximo regulado por el ministerio en la Resolución 4313 de 2011 (Tabla 2).

Para la valoración de los procedimientos se realizó una encuesta a varias aseguradoras, que concentran $72 \%$ de la población afiliada en Colombia, para determinar cuál era el manual tarifario y las tarifas de negociación más empleadas para contratar con proveedores. A partir de esta consulta se determinó que los diferentes manuales y tarifas de contrata- ción se pueden equiparar a los valores del Manual Tarifario del Instituto de Seguros Sociales, ISS 2001 más 30\%, con un porcentaje mínimo de $+25 \%$ y máximo de $+48 \%$. Por último, para los insumos se empleó información suministrada por un hospital de tercer nivel de la ciudad de Medellín.

En el caso base se asumió un horizonte temporal de 10 años, aunque también se presentan los resultados a uno y cinco años. Se emplea una tasa de descuento de 3\%, tanto para costos como para la efectividad, según las recomendaciones de la Guía Metodológica Colombiana (12).

Al comparar los costos y resultados de las dos tecnologías pueden surgir cuatro escenarios (13):

1) prasugrel es más costoso y menos efectivo que el clopidogrel, en cuyo caso decimos que es una estrategia "dominada" y la decisión es no aceptarlo; 2) es menos costoso y más efectivo, es decir, es una estrategia "dominante", lo que implica que la decisión óptima es aceptar esta nueva tecnología; 3) el prasugrel es menos costoso y menos efectivo que el clopidogrel o 4) es más costoso y más efectivo.

En los dos últimos casos se calcula la relación incremental de costo-efectividad (RICE), así:

Relación de costo efectividad incremental $=\frac{\Delta C}{\Delta E}=\frac{\left(C_{i}-C_{j}\right)}{\left(E_{i}-E_{j}\right)}$

donde el numerador representa el costo incremental de la alternativa $i$ comparada con una alternativa $j$ y el denominador representa la efectividad incremental en la unidad seleccionada. Esta razón indica el costo en el que se incurre por cada unidad adicional de ganancia en efectividad.

Asumiendo que el Ministerio de Salud de Colombia enfrenta un presupuesto establecido de manera exógena y persigue unos objetivos específicos; la relación incremental de costo efectividad de una tecnología se puede comparar con un umbral $(\lambda)$ que representa el costo de oportunidad en términos de salud, de desplazar otros programas y servicios, con el fin de liberar los recursos necesarios para financiar la nueva tecnología. Una nueva intervención se considera costo efectiva si los beneficios en salud de la nueva intervención son mayores que los beneficios en salud perdidos como consecuencia del desplazamiento de otras intervenciones, o en otras palabras, si la RICE es menor que $\lambda$ (14).

En Colombia no se ha definido un umbral de manera explícita y no es objeto de esta evaluación realizar una estimación de la disposición a pagar por resultados en salud.

Tabla 2. Precio unitario de los medicamentos.

\begin{tabular}{|l|c|c|c|}
\hline Antiagregantes & Unidad & Precio unitario & Costo total anual \\
\hline Clopidogrel & $\mathrm{Mg}$ & $\$ 34.38$ & $\$ 948796$ \\
Prasugrel & $\mathrm{Mg}$ & $\$ 720.65$ & $\$ 2666388$ \\
Clopidogrel* & $\mathrm{Mg}$ & $\$ 71.83$ & $\$ 1982508$ \\
\hline * Precio máximo según Resolución 4316 de 2011) \\
\hline
\end{tabular}


Sin embargo, para interpretar los resultados en el caso que sea necesario calcular la relación incremental, seguiremos las recomendaciones de la Organización Mundial de la Salud, las cuales establecen que una intervención puede denominarse como costo-efectiva si la relación incremental de costo efectividad es inferior a tres veces el valor del producto interno bruto, PIB per-cápita; que para el 2010 en Colombia corresponde a $\$ 36143349$ pesos.

Se realizó un análisis de sensibilidad determinístico respecto al precio de clopidogrel, el cual se modificó de acuerdo con el precio de recobro máximo estipulado por el gobierno de Colombia (Resolución 4316 de 2011). La tasa de descuento se modificó en 0 y $5 \%$, según las recomendaciones de la Guía Metodológica Colombiana (15).

Además, se construyó un análisis de sensibilidad probabilístico, en donde los costos para cada uno de los desenlaces se representaron mediante distribuciones triangulares (función de distribución continua que se emplea en situaciones en las cuales no se dispone de mucha información respecto a la distribución de las variables). Para especificarlas es necesario definir tres valores: el mínimo esperado, la moda y el valor máximo esperado. Para estimar cada uno de estos valores, se consideraron los diferentes porcentajes de contratación mencionados anteriormente y el tipo de stent empleado (Tabla 3).

Para las probabilidades de transición y ponderaciones de utilidad se empleó la distribución Beta, cuyo rango es $[0,1]$ y por lo tanto es adecuada para representar estas variables. En el análisis probabilístico se realizaron 10000 simulaciones de Monte Carlo y los resultados se presentaron mediante curvas de aceptabilidad. Estas representan la probabilidad de que una intervención sea costo efectiva para distintos valores del umbral de disposición a pagar. Los cálculos en el caso base y todos los análisis de sensibilidad determinísticos y probabilísticos se realizaron en el software TreeAge Pro 2009®.

\section{Resultados}

En el caso base y con un horizonte temporal de 10 años, prasugrel genera un costo adicional de \$1937949 y un incremento de 0.024 años de vida ajustados por calidad (AVAC) por paciente, lo cual implica un costo por AVAC ganado de $\$ 79987$ 695. El resultado es sensible a cambios en el horizonte temporal, ya que se observa que a medida en que se considera un horizonte temporal más grande, la RICE aumenta sustancialmente hasta llegar a ser \$1 490614 891, si sólo se tiene en cuenta el costo y la efectividad durante el primer año (Tabla 4).

Con estos resultados, se observa que para todos los horizontes temporales considerados, la RICE es mayor que el umbral usado en este estudio, por lo que prasugrel no sería una estrategia costo efectiva respecto al tratamiento con clopidogrel.

Por otro lado, se encuentra que los resultados son sensibles al precio del clopidogrel. Asumiendo un horizonte temporal de 10 años, se observa que el costo por AVAC ganado desciende a $\$ 11321457$, si para el cálculo del costo anual de tratamiento con clopidogrel se tiene en cuenta el costo máximo regulado (Tabla 5). De esta manera, si el precio por miligramo de clopidogrel se ubica como el ponderado de sus presentaciones en el mercado, prasugrel no sería costo efectivo. Sin embargo, si el precio se ubicara en el máximo permitido por la regulación del país, la RICE sería menor que el umbral considerado aquí, por lo que prasugrel sí sería costo efectivo.

Tabla 3. Costos de los desenlaces considerados en los modelos.

\begin{tabular}{|c|c|c|c|}
\hline Desenlace & $\begin{array}{c}\text { Mínimo } \\
\text { (Tarifas ISS+25\%, stent convencional) }\end{array}$ & $\begin{array}{c}\text { Moda } \\
\text { (Tarifas ISS }+\mathbf{3 0} \% \text {, stent convencional) }\end{array}$ & $\begin{array}{c}\text { Máximo } \\
\text { (Tarifas ISS+48\%, stent medicado) }\end{array}$ \\
\hline IM & $\$ 8431277$ & $\$ 8579284$ & $\$ 12878934$ \\
\hline Costo programa rehabilitación cardiaca & $\$ 815906$ & $\$ 848543$ & $\$ 966033$ \\
\hline $\mathrm{ACV}$ & $\$ 2143076$ & $\$ 2219670$ & $\$ 2495412$ \\
\hline Pos ACV & $\$ 335076$ & $\$ 346221$ & $\$ 386343$ \\
\hline Sangrado mayor & $\$ 615475$ & $\$ 640094$ & $\$ 728722$ \\
\hline
\end{tabular}

Tabla 4. Análisis de sensibilidad: horizonte temporal.

\begin{tabular}{|c|c|c|c|c|c|c|c|}
\hline $\begin{array}{l}\text { Horizonte } \\
\text { Temporal }\end{array}$ & Estrategia & $\begin{array}{c}\text { Costo } \\
\text { por paciente }\end{array}$ & $\begin{array}{c}\text { Costo } \\
\text { incremental }\end{array}$ & Efectividad & $\begin{array}{l}\text { Efectividad } \\
\text { incremental }\end{array}$ & $\begin{array}{c}\text { Costo/ } \\
\text { Efectividad }\end{array}$ & RICE \\
\hline 1 año & $\begin{array}{c}\text { clopidogrel } \\
\text { prasugrel }\end{array}$ & $\begin{array}{l}\$ 1972136 \\
\$ 3460128\end{array}$ & $\$ 1487992$ & $\begin{array}{l}0.818 \text { avac } \\
0.819 \text { avac }\end{array}$ & $0.001 \mathrm{avac}$ & $\begin{array}{l}\$ 2411909 \\
\$ 4226554\end{array}$ & $\$ 1490614891$ \\
\hline 5 años & $\begin{array}{c}\text { clopidogrel } \\
\text { prasugrel }\end{array}$ & $\begin{array}{l}\$ 2718418 \\
\$ 4511065\end{array}$ & $\$ 1792647$ & $\begin{array}{l}4.434 \text { avac } \\
4.449 \text { avac }\end{array}$ & $0.015 \mathrm{avac}$ & $\begin{array}{c}\$ 613063 \\
\$ 1013847\end{array}$ & $\$ 117218848$ \\
\hline 10 años & $\begin{array}{c}\text { clopidogrel } \\
\text { prasugrel }\end{array}$ & $\begin{array}{l}\$ 3408401 \\
\$ 5346350\end{array}$ & $\$ 1937949$ & $\begin{array}{l}7.336 \text { avac } \\
7.360 \text { avac }\end{array}$ & 0.024 avac & $\begin{array}{l}\$ 464617 \\
\$ 726389\end{array}$ & $\$ 79987695$ \\
\hline
\end{tabular}


Adicionalmente, se encuentra que los resultados no varían significativamente si se modifica la tasa de descuento. Para un horizonte temporal de 10 años se observa que la RICE sería de $\$ 72192589$ si no se aplica tasa de descuento y de \$85 308457 si se utiliza una de 5\% (Tabla 6).

Por último, se evidencia que la inclusión en el modelo de la posibilidad de sufrir nuevos eventos cardiovasculares en el largo plazo no modifica significativamente los resultados. En la Tabla 7 se presentan los resultados de este modelo para un horizonte temporal de cinco y 10 años.

En el análisis de sensibilidad probabilístico (ver resultados de las simulaciones en la Figura 2) se observa que prasugrel, por lo general implica mayores costos comparado con el clopidogrel con una efectividad similar. Además, en la Figura 3 se presenta la curva de aceptabilidad, en donde se encuentra que para el umbral considerado en este trabajo, la probabilidad de que prasugrel sea una estrategia costo efectiva es cercana a $7 \%$.

\section{Discusión}

Los resultados de este análisis económico sugieren que la relación de costo efectividad de prasugrel depende significativamente del precio del clopidogrel que el decisor considere adecuado. Si éste se ubica en el precio estimado a partir de la base de datos del SISMED, en ninguno de los escenarios considerados en este estudio, prasugrel se considera una estrategia costo efectiva (su RICE es mayor que $\lambda$ ). Sin embargo, si el precio del clopidogrel se ubica en el máximo permitido por la regulación del país, prasugrel sería una estrategia costo efectiva. Por lo anterior, es fundamental determinar el costo real asumido por el sistema con el fin de

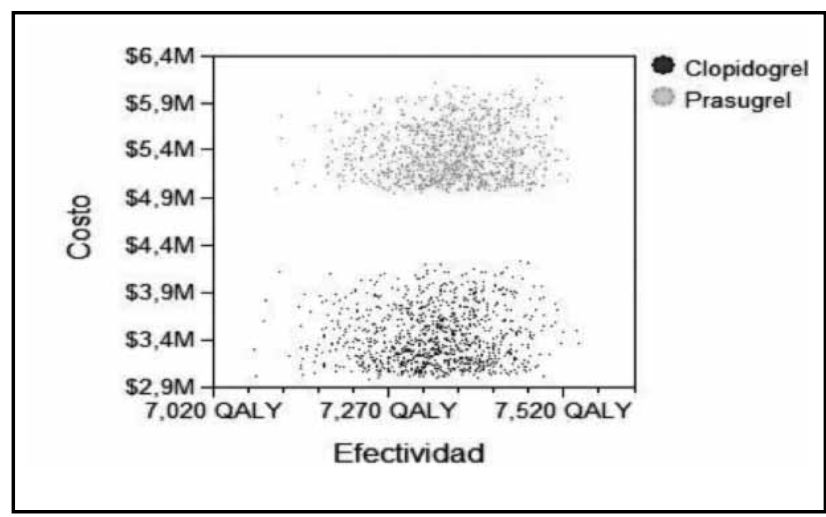

Figura 2. Gráfico de dispersión.

Tabla 5. Análisis de sensibilidad: precio del clopidogrel.

\begin{tabular}{|c|c|c|c|c|c|c|c|}
\hline $\begin{array}{c}\text { Precio del } \\
\text { Clopidogrel }\end{array}$ & Estrategia & $\begin{array}{l}\text { Costo por } \\
\text { paciente }\end{array}$ & $\begin{array}{c}\text { Costo } \\
\text { incremental }\end{array}$ & Efectividad & $\begin{array}{l}\text { Efectividad } \\
\text { incremental }\end{array}$ & Costo/Efectividad & RICE \\
\hline$\$ 948796$ & $\begin{array}{c}\text { Clopidogrel } \\
\text { Prasugrel }\end{array}$ & $\begin{array}{l}\$ 3408401 \\
\$ 5346350\end{array}$ & $\$ 1937949$ & $\begin{array}{l}7.336 \text { AVAC } \\
7.360 \text { AVAC }\end{array}$ & 0.024 AVAC & $\begin{array}{l}\$ 464617 \\
\$ 726389\end{array}$ & $\$ 79987695$ \\
\hline$\$ 1982508$ & $\begin{array}{c}\text { Clopidogrel } \\
\text { Prasugrel }\end{array}$ & $\begin{array}{l}\$ 4631927 \\
\$ 5346350\end{array}$ & $\$ 714423$ & $\begin{array}{l}7.336 \text { AVAC } \\
7.360 \text { AVAC }\end{array}$ & 0.024 AVAC & $\begin{array}{l}\$ 631402 \\
\$ 726389\end{array}$ & $\$ 29487404$ \\
\hline
\end{tabular}

Tabla 6. Análisis de sensibilidad: tasa de descuento.

\begin{tabular}{|c|c|c|c|c|c|c|c|}
\hline $\begin{array}{c}\text { Tasa de } \\
\text { descuento }\end{array}$ & Estrategia & $\begin{array}{l}\text { Costo por } \\
\text { paciente }\end{array}$ & $\begin{array}{c}\text { Costo } \\
\text { incremental }\end{array}$ & Efectividad & $\begin{array}{l}\text { Efectividad } \\
\text { incremental }\end{array}$ & Costo/Efectividad & RICE \\
\hline $\mathbf{0}$ & $\begin{array}{c}\text { Clopidogrel } \\
\text { Prasugrel }\end{array}$ & $\begin{array}{l}\$ 3683380 \\
\$ 5679242\end{array}$ & $\$ 1995861$ & $\begin{array}{l}\text { 8.432 AVAC } \\
8.460 \text { AVAC }\end{array}$ & 0.028 AVAC & $\begin{array}{l}\$ 436825 \\
\$ 671320\end{array}$ & $\$ 72192589$ \\
\hline 0.05 & $\begin{array}{c}\text { Clopidogrel } \\
\text { Prasugrel }\end{array}$ & $\begin{array}{l}\$ 3255697 \\
\$ 5161485\end{array}$ & $\$ 1905788$ & $\begin{array}{l}\text { 6.732 AVAC } \\
6.754 \text { AVAC }\end{array}$ & 0.022 AVAC & $\begin{array}{l}\$ 483650 \\
\$ 764228\end{array}$ & $\$ 85308457$ \\
\hline
\end{tabular}

Tabla 7. Análisis de sensibilidad: Consideración de nuevos eventos cardiovasculares en el largo plazo.

\begin{tabular}{|c|c|c|c|c|c|c|c|}
\hline $\begin{array}{l}\text { Horizonte } \\
\text { temporal }\end{array}$ & Estrategia & $\begin{array}{l}\text { Costo por } \\
\text { paciente }\end{array}$ & $\begin{array}{c}\text { Costo } \\
\text { incremental }\end{array}$ & Efectividad & $\begin{array}{l}\text { Efectividad } \\
\text { incremental }\end{array}$ & Costo/Efectividad & RICE \\
\hline 5 años & $\begin{array}{c}\text { Clopidogrel } \\
\text { Prasugrel }\end{array}$ & $\begin{array}{l}\$ 2772316 \\
\$ 4567991\end{array}$ & $\$ 1795675$ & $\begin{array}{l}\text { 4.433 AVAC } \\
4.448 \text { AVAC }\end{array}$ & 0.015 AVAC & $\begin{array}{c}\$ 625382 \\
\$ 1026877\end{array}$ & $\$ 116352201$ \\
\hline 10 años & $\begin{array}{c}\text { Clopidogrel } \\
\text { Prasugrel }\end{array}$ & $\begin{array}{l}\$ 3485455 \\
\$ 5431286\end{array}$ & $\$ 1945832$ & $\begin{array}{l}7.334 \text { AVAC } \\
7.358 \text { AVAC }\end{array}$ & 0.024 AVAC & $\begin{array}{c}\$ 47266 \\
\$ 738138\end{array}$ & $\$ 79753744$ \\
\hline
\end{tabular}




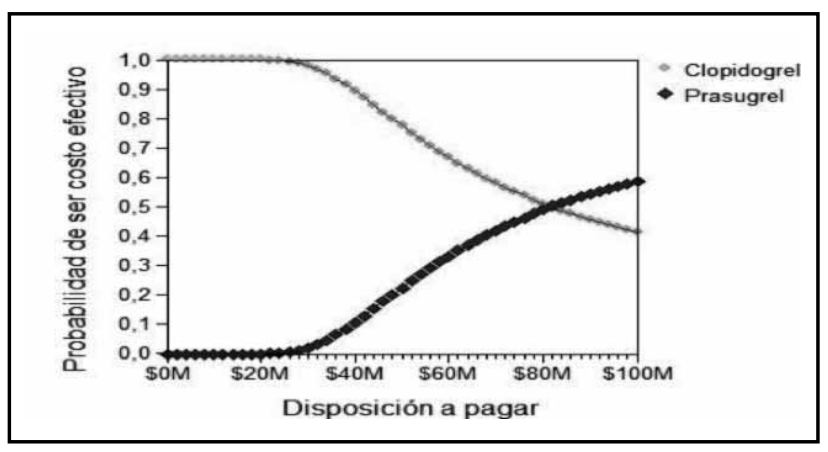

Figura 3. Curva de aceptabilidad.

establecer el valor más adecuado para tomar una decisión, pues la diferencia entre el precio estimado a partir de la base de datos del SISMED y el precio regulado es superior a $50 \%$.

Otra variable que modifica sustancialmente los resultados, es el horizonte temporal, ya que limitar la evaluación a un horizonte de corto plazo puede subestimar los costos y beneficios de las alternativas que se comparan y por lo tanto conducir a conclusiones erróneas sobre el costo-efectividad de las nuevas tecnologías. Por tal motivo en este estudio se adoptó un horizonte temporal lo suficientemente amplio para capturar el efecto de las tecnologías sobre los costos y el estado de salud en el largo plazo, el cual puede ser más relevante para el decisor. Sin embargo, incluso en un horizonte temporal de largo plazo, la RICE sigue estando por debajo del umbral (si se considera el precio del clopidogrel como el estimado a partir del SISMED).

En este análisis se trató de incorporar la mejor evidencia disponible respecto a la efectividad de las intervenciones con el fin de apoyar las recomendaciones clínicas. Se consideraron los resultados del ensayo clínico más importante que ha comparado ambos medicamentos así como resultados de estudios realizados en Colombia. Mediante el modelo de Markov se estimó el impacto esperado de las intervenciones en el largo plazo con el fin de abarcar todos los costos y consecuencias que implican las intervenciones y que pueden ser relevantes para tomar una decisión.

Las conclusiones encontradas aquí difieren de las evaluaciones económicas realizadas en Estados Unidos y el Reino Unido. Por una parte, NICE (16) encuentra que para pacientes con SCA sometidos a una ICP en el Reino Unido, prasugrel genera un costo adicional de $£ 3220$ por AVAC ganado en comparación con clopidogrel. Además, considerando un umbral de $£ 20000$ por AVAC ganado, encuentran la probabilidad de que prasugrel sea costo efectivo para toda la población varía entre 75 y $80 \%$, y para pacientes diabéticos llega a ser casi de $100 \%$. Por otro lado, Mahoney et al (17) concluyen que en Estados Unidos, para pacientes con SCA que se someten a ICP, prasugrel es una estrategia altamente costo efectiva y en muchos escenarios menos costosa que el clopidogrel. Las diferencias respecto en los resultados encontrados en este trabajo, se pueden deber a las diferencias en el precio del medicamento, y a que en Colombia se tienen menores disposiciones a pagar que en estos países, los cuales son más desarrollados socioeconómicamente y tienen más recursos disponibles en sus sistemas de salud.

En el estudio se reconocen varias limitaciones: en primer lugar, los datos de efectividad, en particular las probabilidades de transición, se tomaron de la información disponible en los artículos publicados, lo cual impide el uso de análisis de supervivencia para realizar estimaciones más precisas. En segundo lugar, las ponderaciones de utilidad para los distintos desenlaces se calcularon a partir de la valoración del EQ-5D realizada en Reino Unido para ticagrelor. Es importante avanzar en la estimación de ponderaciones de utilidad para desenlaces en salud en Colombia, sí existe la voluntad política de informar las decisiones en salud por análisis económicos.

\section{Financiación}

Este artículo se elaboró como parte del desarrollo de la Guía de Atención Integral basada en la evidencia, para la detección temprana, atención integral, seguimiento y rehabilitación de pacientes con diagnóstico de un evento coronario agudo, la cual estuvo a cargo de la Universidad de Antioquia, en asocio con la Universidad Nacional, sede Bogotá, y la Pontificia Universidad Javeriana, sede Bogotá, y financiada por Colciencias y el Ministerio de Salud y Protección Social.

\section{Conflictos de interés}

Juan Manuel Senior fue presidente de la Asociación Colombiana de Medicina Interna y partícipe de la realización del estudio multicéntrico internacional Trilogy ACS.

\section{Agradecimientos}

Se agradece a todo el grupo desarrollador de la Guía de Atención Integral basada en la evidencia, para la detección temprana, atención integral, seguimiento y rehabilitación de pacientes con diagnóstico de un evento coronario agudo, el cual estuvo conformado, además de los autores, por: Luz Helena Lugo, Óscar Ossio, James Díaz, Jorge Luis Acosta, Jesús Alberto Plata, Erick Trespalacios, Natalia Acosta, Álvaro Quintero, Pilar Pastor, Manuel Urina, Ricardo Bohórquez, Sebastián Vélez, Eduardo Ramírez, Fernán del Cristo Mendoza, Juan José Vélez, Paula Andrea Castro, Paola Andrea Ramírez y Ángela Orozco.

\section{Referencias}

1. Senior JM. Síndrome coronario agudo. In: Archila PE, Senior JM, editors. Texto de medicina interna : aprendizaje basado en problemas. Libro oficial de la ACMI (Asociación Colombiana de Medicina Interna). Bogotá: Distribuna; 2013.

2. DANE. Departamento Nacional de Estadística. Estadísticas vitales. Bogotá: DANE; 2012.

3. Karnon J, Bakhai A, Brennan A, Pandor A, Flather M, Warren E, et al. A cost-utility analysis of clopidogrel in patients with non-ST-segment-elevation acute coronary syndromes in the UK. Int J Cardiol. 2006; 109 (3): 307-16.

4. Main C, Palmer S, Griffin S, Jones L, Orton V, Sculpher M, et al. Clopidogrel used in combination with aspirin compared with aspirin alone in the treatment of non-ST-segment-elevation acute coronary syndromes: a systematic review and economic evaluation. Health Technol Assess. 2004; 8(40): iii-iv, xv-xvi, 1-141.

5. Berg J, Lindgren P, Spiesser J, Parry D, Jonsson B. Cost-effectiveness of clopidogrel in myocardial infarction with ST-segment elevation: a European model based on the CLARITY and COMMIT trials. Clin Ther. 2007; 29 (6): 1184-202.

6. Berg J, Fidan D, Lindgren P. Cost-effectiveness of clopidogrel treatment in percutaneous coronary intervention: a European model based on a meta-analysis of the PCI-CURE, CREDO and PCI-CLARITY trials. Curr Med Res Opin. 2008; 24 (7): 2089-101.

7. Yusuf S, Zhao F, Mehta SR, Chrolavicius S, Tognoni G, Fox KK, et al. Effects of clopidogrel in addition to aspirin in patients with acute coronary syndromes without ST-segment elevation. N Engl J Med. 2001; 345 (7): 494-502.

8. Sabatine MS, Cannon CP, Gibson CM, Lopez-Sendon JL, Montalescot G, Theroux P, et al. Addition of clopidogrel to aspirin and fibrinolytic therapy for myocardial infarction with ST-segment elevation. N Engl J Med. 2005; 352 (12): 1179-89. 
9. Astra Zeneca. Ticagrelor for the treatment of Acute Coronary Syndromes. NICE submission. Sing Techn Appra. 2010.

10. Wiviott SD, Braunwald E, McCabe CH, Montalescot G, Ruzyllo W, Gottlieb $\mathbf{S}$, et al. Prasugrel versus clopidogrel in patients with acute coronary syndromes. N Engl J Med. 2007; 357 (20): 2001-15.

11. Ministerio de la Protección Social de Colombia. Sistema de información de precios de medicamentos SISMED. Bogotá: Ministerio de la Protección Social; 2012.

12. Ministerio de la Protección Social de Colombia, Colciencias. Guía Metodológica para la elaboración de Guías Atención Integral en el Sistema General de Seguridad Social en Salud Colombiano. Bogotá 2010. Available from: http://www.minsalud. gov.co/salud/Documents/Gu\%C3\%ADa\%20Metodol\%C3\%B3gica\%20para\%20 la\%20elaboraci\%C3\%B3n\%20de\%20gu\%C3\%ADas.pdf.

13.Johannesson M, Weinstein MC. On the decision rules of cost-effectiveness analysis. J Health Econ. 1993; 12 (4): 459-67.
14. Claxton K, Walker S, Palmer S, Sculpher M. Appropriate Perspectives for Health Care Decisions. Working Papers. 2010 (054 cherp).

15. Ministerio de la Protección Social de Colombia, Colciencias. Guía Metodológica para la elaboración de Guías Atención Integral en el Sistema General de Seguridad Social en Salud Colombiano. Bogotá 2010. Available from: http://www.minsalud. gov.co/salud/Documents/Gu\%C3\%ADa\%20Metodol\%C3\%B3gica\%20para\%20 la\%20elaboraci\%C3\%B3n\%20de\%20gu\%C3\%ADas.pdf.

16. National Institute for Health and Clinical Excellence. Prasugrel for the treatment of acute coronary syndromes with percutaneous coronary intervention. London: NICE; 2009-2010.

17. Mahoney EM, Wang K, Arnold SV, Proskorovsky I, Wiviott S, Antman E, et al. Cost-effectiveness of prasugrel versus clopidogrel in patients with acute coronary syndromes and planned percutaneous coronary intervention: results from the trial to assess improvement in therapeutic outcomes by optimizing platelet inhibition with Prasugrel-Thrombolysis in Myocardial Infarction TRITON-TIMI 38. Circulation. 2010; 121 (1): 71-9. 